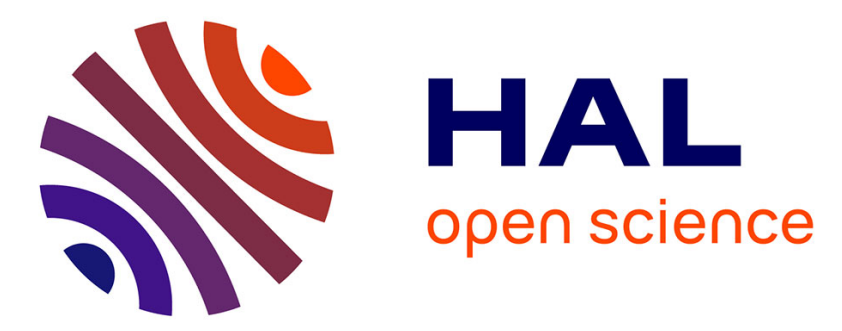

\title{
Fusion of spatial relationships for guiding recognition, example of brain structure recognition in 3D MRI
}

\author{
Isabelle Bloch, Olivier Colliot, Oscar Camara, Thierry Géraud
}

\section{To cite this version:}

Isabelle Bloch, Olivier Colliot, Oscar Camara, Thierry Géraud. Fusion of spatial relationships for guiding recognition, example of brain structure recognition in 3D MRI. Pattern Recognition Letters, 2005, 26 (4), pp.449-457. 10.1016/j.patrec.2004.08.009 • hal-01251245

\section{HAL Id: hal-01251245 \\ https://inria.hal.science/hal-01251245}

Submitted on 5 Jan 2016

HAL is a multi-disciplinary open access archive for the deposit and dissemination of scientific research documents, whether they are published or not. The documents may come from teaching and research institutions in France or abroad, or from public or private research centers.
L'archive ouverte pluridisciplinaire HAL, est destinée au dépôt et à la diffusion de documents scientifiques de niveau recherche, publiés ou non, émanant des établissements d'enseignement et de recherche français ou étrangers, des laboratoires publics ou privés. 


\title{
Fusion of Spatial Relationships for Guiding Recognition, Example of Brain Structure Recognition in 3D MRI
}

\author{
Isabelle Bloch ${ }^{\mathrm{a}}$, Olivier Colliot ${ }^{\mathrm{b}}$, Oscar Camara ${ }^{\mathrm{c}}$, and \\ Thierry Géraud ${ }^{\mathrm{d}}$ \\ ${ }^{a}$ Ecole Nationale Supérieure des Télécommunications, Dept. TSI, CNRS UMR \\ 5141 LTCI, 46 rue Barrault, 75013 Paris, France - Isabelle.Bloch@enst.fr \\ ${ }^{\mathrm{b}}$ McConnell Brain Imaging Center, MNI, McGill University, 3801 University, \\ Montréal, Québec, H3A2B4, Canada-colliot@bic.mni.mcgill.ca \\ ${ }^{\mathrm{c}}$ King's College, London, UK - Oscar.Camara@kcl.ac.uk \\ ${ }^{\mathrm{d}}$ EPITA Research and Development Laboratory, 14-16 rue Voltaire, F-94276 Le \\ Kremlin-Bicêtre cedex, France - thierry.geraud@lrde.epita.fr
}

\begin{abstract}
Spatial relations play an important role in recognition of structures embedded in a complex environment and for reasoning under imprecision. Several types of relationships can be modeled in a unified way using fuzzy mathematical morphology. Their combination benefits from the powerful framework of fuzzy set theory for fusion tasks and decision making. This paper presents several methods of fusion of information about spatial relationships and illustrates them on the example of model-based recognition of brain structures in 3D magnetic resonance imaging.
\end{abstract}

Key words: Spatial relationships, fuzzy mathematical morphology, knowledge representation, information fusion, model-based recognition, brain imaging.

\section{Introduction}

The aim of this paper is to emphasize the role of spatial relationships and of their fusion in structural pattern recognition in images. The proposed methodology is illustrated in a $3 \mathrm{D}$ brain imaging application.

When several objects or structures have to be recognized in an image, it happens in several applications that characteristics of the objects themselves may not be discriminating enough to achieve an individual recognition. In such 
cases, spatial relationships between objects become of prime importance for recognition, as a complementary information. An example, that will be further detailed in Section 4, concerns the recognition of internal brain structures in 3D magnetic resonance images (MRI). Internal grey nuclei all have similar grey levels and shapes that are subject to individual variability and therefore object characteristics are not discriminating enough. Spatial relationships can solve ambiguities by providing a structural information on the scene, through the description of the spatial arrangement of the objects (prior anatomical knowledge), and thus guide recognition. Moreover such relationships are much less prone to variability than shape, size or grey levels.

Several types of spatial relationships must usually be considered to assess the structure of a given scene. According to the semantic hierarchy proposed in [1], we consider two main classes of relationships (corresponding to levels 3 and 4 of this hierarchy): topological ones, which include part-whole relationships such as inclusion, exclusion, adjacency, etc.; and metric relationships such as distances and orientations. Therefore, the recognition process can be guided by the combination of several of these relations, which requires information fusion tools $[2,3]$.

The fuzzy set and possibility theory framework has many interesting features to address these issues. Indeed it provides appropriate tools for representing spatial imprecision, imprecision in knowledge description (useful in knowledge representation), to define vague relationships, and it offers a large set of fusion operators and decision making tools [4-6]. We recognize in these features the

main ingredients of a recognition system. Therefore the work presented in this paper relies on this framework ${ }^{1}$.

In Section 2, we briefly address the knowledge representation issue. Then, the basic fusion tools are summarized in Section 3, and we propose several methods to include fusion in recognition procedures. A novel application of model-based recognition based on fusion of spatial relationships is presented in Section 4.

\section{Knowledge representation: modeling spatial relationships in the fuzzy set framework}

The fuzzy set framework is interesting for modeling spatial relationships for several reasons:

- the objects of interest can be imprecisely defined, for instance due to pre-

$\overline{1}$ This paper extends the work presented in [7]. 
viously applied image processing steps such as segmentation;

- some relations are imprecise, such as to be left of, and find a more suitable definition in the fuzzy set framework;

- the type of knowledge available about the structures or the type of question we would like to answer can be imprecise too.

We consider set theoretical relations, adjacency, distances, and directional relative position, which are the main binary spatial relationships. Some of them have led to a rich literature in the fuzzy set community, like distances which have been defined by means of several different approaches, while other relations have not raised so much attention. Our work in this domain was mainly based on fuzzy mathematical morphology [8], which allows us to represent in a unified way various spatial relationships [9].

Two types of questions are important for applications in structural pattern recognition:

(1) given two objects (possibly fuzzy), assess the degree to which a relation is satisfied;

(2) given one reference object, define the area of the space in which a relation to this reference is satisfied (to some degree).

Our approach provides answers to these two types of questions.

Since the representation issues where already addressed in previous papers (see e.g. [9-11] and the references therein), they are not detailed here. This paper focuses on the subsequent steps of fusion and decision making in recognition problems. We just recall the underlying principle: the main idea to extend relations on crisp objects to fuzzy objects is to translate crisp equations into fuzzy ones by using fuzzy equivalents of logical and set theoretical operators (for instance an intersection is translated as a t-norm, etc.). Let us give an example: defining the region of the space where an object can be searched, knowing that it is at a distance between $n_{1}$ and $n_{2}$ from an object $A$, can be performed by computing $D^{n_{2}}(A) \backslash D^{n_{1}-1}(A)$, where $D^{n}$ denotes the dilation of size $n$ (this is obtained by exploiting links between minimum and Hausdorff distances and morphological dilation). If the distance interval is defined as a fuzzy interval, two fuzzy structuring elements are defined and fuzzy dilation is applied. In more difficult cases where the relationship is intrinsically vague (such as directional relationships: "left of", etc.), it is better to propose directly a fuzzy definition, even for crisp objects. Dilation by a fuzzy directional structuring element, representing spatially the semantics of the considered direction, proved to be a useful tool, leading to good properties and behaviors. 


\section{Fusion}

Fusion operators and their choice For the combination step in the fusion process, the advantages of fuzzy sets lie in the variety of combination operators, which may deal with heterogeneous information $[12,13,3]$. We proposed a classification of these operators with respect to their behavior (in terms of conjunctive, disjunctive, and compromise [12]), the possible control of this behavior, their properties and their decisiveness, which proved to be useful for several applications in image processing [2].

Operators such as t-norms, t-conorms and mean operators always behave respectively in a conjunctive, disjunctive and compromise way. Within each class, some operators are more severe or more indulgent, some are more or less discriminating, etc. Operators such as symmetrical sums behave differently based on the values to be combined. Other operators depend on additional information such as conflict, reliability, context, etc.

It is of particular interest to note that, unlike other data fusion theories (like Bayesian or Dempster-Shafer combination), fuzzy sets provide a great flexibility in the choice of the operator, that can be adapted to any situation at hand. This choice can be guided by the proposed classification. Indeed, one often has to deal with situations where a piece of information is reliable only for some structures, or is not able to discriminate between two objects while another piece of information does. In this context, some operators are particularly powerful, like operators that behave differently depending on whether the values to be combined are of the same order of magnitude or not, whether they are small or high, and operators that depend on some global knowledge.

A noticeable advantage of this approach is that it is able to combine heterogeneous information, like it is the case here, where several types of knowledge and information with different semantics have to be combined, and it avoids defining a more or less arbitrary and questionable metric between pieces of information.

Let us give a few examples. If we have different constraints about an object (for instance concerning the relations it should have with respect to another object) which have all to be satisfied, these constraints can be combined using a t-norm (a conjunction). This is typically the case when an object is described using relations to several objects or several relations of different types to the same object.

If one object has to satisfy one relation or another one then a disjunction represented by a t-conorm has to be used. This occurs for instance when two symmetrical structures with respect to the reference object can be found (e.g. in the brain where a high degree of symmetry exists between structures in 
both hemispheres).

Mean operators can be used to combine several estimations and try to find a compromise between them. Such operators have a compensation effect which is interesting in cases where both under-estimation and over-estimation occur.

Operators with a variable behavior may also be of great interest. For instance associative symmetrical sums can be used for reinforcing the dynamics between high and low membership degrees, which has advantages for the decision step (since a better discrimination between different situations is achieved). Importance of a constraint or reliability factors can be easily introduced in adaptive operators, etc. Further examples will be given below.

Including fusion in recognition procedures A first recognition approach, called global approach, uses the first type of question raised in Section 2. The idea is to represent all available knowledge about the objects to be recognized. In the case of model-based recognition, two representations are built, one for the model and one for the data, and recognition is then expressed as a comparison between both representations. A typical example consists of graph-based representations. The model is then represented as a graph where nodes are objects and edges represent links between these objects. Both nodes and edges are attributed. Node attributes are characteristics of the objects, while edge attributes quantify spatial relationships between the objects. A data graph is then constructed from each image where the recognition has to be performed. Each region of the image (obtained after some processing) constitutes a node of this data graph, and edges represent spatial relationships between regions, as for the model graph.

The comparison between representations is performed through the computation of similarities between model graph attributes and data graph attributes. The fusion takes mainly place at this level, in order to combine the similarity values for different relationships. The fusion results constitute an objective function to be optimized by a matching procedure.

Mainly weighted average operators are used for the fusion. Such operators allow to weight differently node attributes and edge attributes, or to give more importance to some relationships than to other ones. This is particularly useful when characteristics of objects or of relations have not the same level of stability and variability. For instance if an attribute corresponds to a highly inter-individual variable feature, a higher dissimilarity can be expected between the model and the image, but should have a low impact on the global objective function.

The similarity is located at an intermediate level, in the sense that it does not 
apply directly to the considered objects but to some global feature extracted from these objects. In order to cope with the summarization aspect of such a feature, it may be interesting to incorporate in the similarity measure a weight representing the quality of the relation. Typically a low confidence should be attached to a relation (like adjacency) between two objects that concerns only a few points. Such confidence values are easy to introduce in weighted operators.

But other operators could be used as well, in order to exploit further the flexibility of the fuzzy set theory. For instance, some symmetrical sums behave in a conjunctive mode for low values and in a disjunctive mode for high values. This results in an increased dynamics in the combined result, which can facilitate the decision process, as mentioned above.

Another aspect is that some relations have not the same impact on recognition depending on whether they are satisfied or not. For instance, the fact that two objects are adjacent like in the model is more relevant to recognition than the fact that they are not adjacent like in the model, and it is then interesting to have a measure that is high only if both values are high [14] (since adjacency occurs between few objects only and is moreover quite sensitive).

A second type of approach relies on the second type of question raised in Section 2 and is called here progressive approach. In such a progressive approach, objects are recognized sequentially and their recognition makes use of knowledge about their relations with respect to other objects. Relations with respect to previously obtained objects can be combined at two different levels of the procedure.

First, fusion can occur in the spatial domain, using spatial fuzzy sets. The result of this fusion allows to build a fuzzy region of interest in which the search of a new object will take place, in a process similar to focalization of attention. In a sequential procedure, the amount of available spatial relations increases with the number of processed objects. Therefore, the recognition of the most difficult structures, usually treated in the last steps, will be focused in a more restricted area. A drawback of this approach may be the possible propagation of errors from one structure to the next one. Therefore, special attention has to be paid to the robustness of each step, hence reinforcing the interest of spatial relations.

Another fusion level occurs during the final decision step: the segmentation of a structure. Instead of only focusing segmentation techniques on a particular area, spatial relations are also used to constrain the segmentation process itself. For this purpose, relations are introduced in the evolution scheme of a deformable model, in which they are combined with other types of numerical information, usually edge and regularity constraints. The evolution of a de- 
formable model can then be described by the following dynamic force equation $[15,16]$ :

$$
\gamma \frac{\partial \mathbf{X}}{\partial t}=\mathbf{F}_{\text {int }}(\mathbf{X})+\mathbf{F}_{e x t}(\mathbf{X})
$$

where $\mathbf{X}$ is the deformable contour or surface, $\mathbf{F}_{\text {int }}$ is the internal force that specifies the regularity of the surface and $\mathbf{F}_{\text {ext }}$ is the external force. Instead of representing only edge information, the external force combines it with spatial relation information. It is then defined as:

$$
\mathbf{F}_{e x t}=\lambda \mathbf{F}_{C}+\nu \mathbf{F}_{R}
$$

where $\mathbf{F}_{C}$ is a classical data term that drives the model towards the edges, $\mathbf{F}_{R}$ is a force associated to spatial relations and $\lambda$ and $\nu$ are weighting coefficients. The role of $\mathbf{F}_{R}$ is to force the deformable model to stay in regions where the relation is fulfilled. In [17], we proposed different methods to compute $\mathbf{F}_{R}$ from a fuzzy set $\mu_{R}$ representing a spatial relation $R$ or a combination of relations obtained at the first fusion level. Common properties of these approaches include the following: the constructed force is directed towards high values of the membership function $\mu_{R}$; it is zero inside the kernel of $\mu_{R}$ and nonzero elsewhere; and its modulus is proportional to $1-\mu_{R}$. As an example, we present here one of these methods which uses the fuzzy set to define an energy potential, for all $x$ in $\mathcal{S}$ :

$$
P_{R}(x)=1-\mu_{R}(x)+d_{\operatorname{supp}(R)}(x)
$$

where $d_{\text {supp }(R)}$ is the distance from the support of $\mu_{R}$ which is used to have a non-zero force outside the support. With the following normalization, we obtain a force satisfying the required properties:

$$
\mathbf{F}_{R}(x)=-\left(1-\mu_{R}(x)\right) \frac{\nabla P_{R}(x)}{\left\|\nabla P_{R}(x)\right\|}
$$

In the following Section and in particular in Figure 3, we will illustrate these equations with an example in brain structures recognition.

\section{An application to the recognition of brain structures based on anatomical knowledge representation}

Let us now illustrate how these fuzzy spatial relations can be used for recognizing structures in a scene based on a model. The chosen example concerns the recognition of internal brain structures (ventricular system and grey nuclei) in 3D MRI. The model is a representation of anatomical knowledge. Different representations can be used, as will be seen below. Two types of approaches are developed (as introduced in Section 3), corresponding to the two types of questions mentioned in Section 2. 


\subsection{Global approach}

In the first approach, spatial relations evaluated between spatial entities (typically objects or regions) are considered as attributes in a graph. The model is a graph derived from an anatomical atlas. Each node represents an anatomical structure, and edges represent spatial relationships between these structures. A data graph is constructed from the MRI image where recognition has to be performed. Each node represents a region obtained from a segmentation method. Since it is difficult to segment directly the objects, usually the graph is based on an over-segmentation of the image, for instance based on watersheds. Attributes are computed as for the model. The use of fuzzy relations is particularly useful in order to be less sensitive to the segmentation step.

One important problem to be solved then is graph matching. Because of the schematic aspect of the model and the difficulty to segment the image into meaningful entities, no isomorphism can be expected between both graphs. In particular, several regions of the image can be assigned to the same node of the model graph. Such problems call for inexact graph matching. In general, it constitutes in finding a morphism, which furthermore optimizes an objective function based on similarities between attributes. Here the fusion applies not directly on the relations but on the similarities between them (see Section 3). A weighted mean operator allows us to give more importance to the edges, which show less variability between subjects and therefore constitute stronger anchors for guiding recognition. The morphism aims at preserving the graph structure, while the objective function privileges the association between nodes, respectively between edges, with similar attribute values. This approach can benefit from the huge literature on fuzzy comparison tools (see e.g. [18]) and from recent developments on fuzzy morphisms [19]. The optimization is not an easy task since the problem is NP-hard. Genetic algorithms, estimation of distribution algorithms (EDA) and tree search methods have been developed towards this aim [20-22].

\subsection{Progressive approach}

Definition of anatomical knowledge In the second type of approach, we use spatial representations of spatial knowledge $[23,17]$. The representation of the model consists of two parts: an iconic part (a digital atlas, Figure 1, left) and a symbolic part (linguistic descriptions of relationships between anatomical structures, as provided by neuro-anatomists). The iconic part is directly a spatial representation, while the linguistic part is translated into spatial fuzzy sets according to the methodology described in [23] (see the summary and the example of distance knowledge representation in Section 2). 
The recognition procedure uses the two levels of fusion described in Section 3. It consists in first recognizing simple structures (typically brain and lateral ventricles), and then progressively more and more difficult structures, based on relationships between these structures and previously recognized ones. Each relationship describing the structure to be recognized is translated into a spatial fuzzy set representing the area satisfying this relation, to some degree. The fuzzy sets representing all relationships involved in the recognition process are combined using a numerical fusion operator.
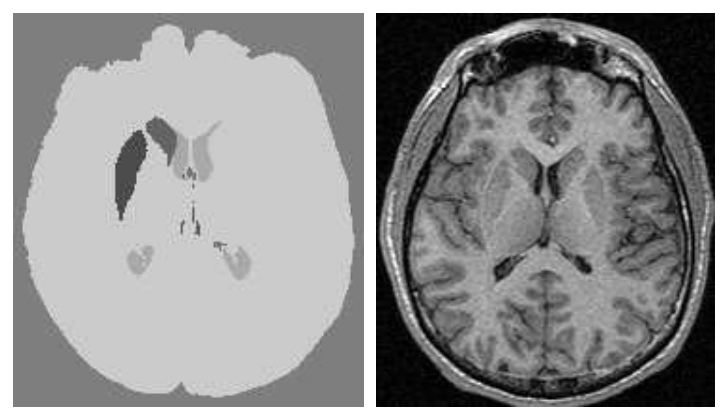

Fig. 1. Slice extracted from a model atlas and from a 3D MRI image. In the atlas, each grey level represents a different object we are interested in.

For instance, the recognition of a caudate nucleus in a 3D MRI image uses the previous recognition of brain and lateral ventricles, the computation of a deformation field between the atlas and the image taking into account these previously recognized objects, and the following pieces of knowledge, as illustrated in Figure 2:

- rough shape and localization are provided by the representation of the caudate nucleus in the atlas, on which a fuzzy dilation is applied to account both for variability and for inexact matching between the model and the image,

- the caudate nucleus belongs to the brain (black) but is outside from both lateral ventricles (white components inside the brain),

- the caudate nucleus is lateral to the lateral ventricle and at a distance less than "about D" from it.

Application of prior knowledge In [23], the localization information provided by the atlas is used as a region of interest. This region is quite restricted which allows an efficient automatic separation of radiometric classes in the region. From the classes obtained in this region, the one that best matches symbolic anatomical knowledge is selected. The fusion is performed in a conjunctive manner for all spatial relations, and using a mean operator for radiometric information.

In [17], we changed the approach in order to avoid the use of the iconic part of the model. This presents two main advantages: the high computation time 


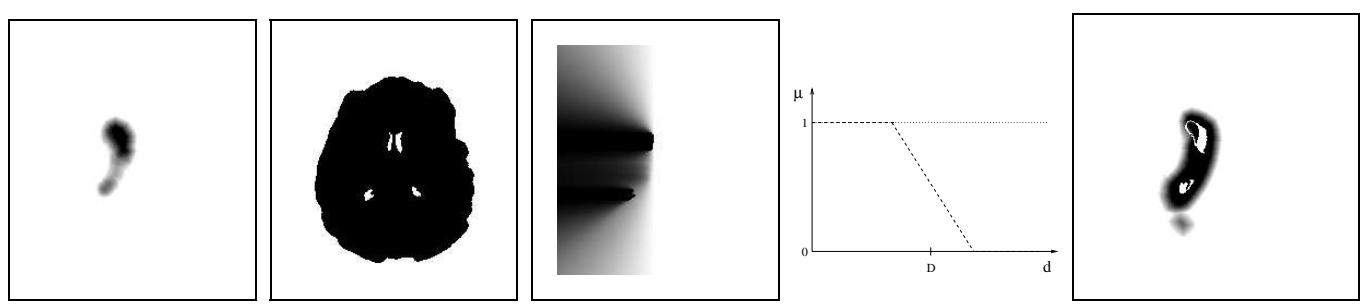

Fig. 2. Information representation in the image space (only one slice of the $3 \mathrm{D}$ volume is shown), illustrating knowledge about one caudate nucleus. From left to right: shape information, set relationships, relative directional relationship, membership function representing distance knowledge, and corresponding spatial fuzzy set (the contours of the caudate nucleus we are looking at are shown in white). Membership values vary from 0 (white) to 1 (black).

associated with the computation of the deformation field is left aside and the procedure is potentially more robust because it uses only the symbolic part of the knowledge, which is generic instead of being built from a single individual as in the iconic atlas. The region of interest, instead of being derived from the atlas, is then defined using the linguistic descriptions, the fusion of spatial relations being still performed using a t-norm. The main drawback is that this region is usually less restricted than the one built with the iconic atlas.

Finally, a refinement stage is introduced using a deformable model. This stage takes the output of the previous classification as a starting point and has the potential to correct possible imperfections of the previous stage together with regularizing the contours of structures. This deformable model makes use of a fusion of heterogeneous knowledge: edge information derived from the image, regularization constraints and spatial relations contained in the linguistic description. All pieces of information are combined using the framework described in Section 3. Figure 3 presents an example of this heterogeneous information, namely the data term and the force corresponding to spatial relations, in the case of the caudate nucleus. Here, the primary role of spatial relations is to prevent the deformable model from progressing beyond the limit of structures with weak boundaries.

Figure 4 shows 3D views of some cerebral objects recognized in an MR image with our method. In particular, the importance of spatial relations is illustrated in the case of the caudate nucleus. The lower part of this structure has a very weakly defined boundary and the use of a spatial relation is essential to achieve a good segmentation. This approach has been tested successfully on more than 10 images. 


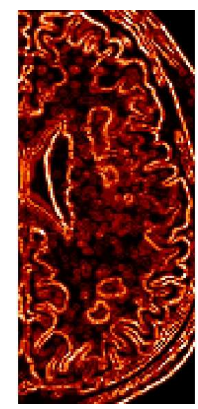

(a)

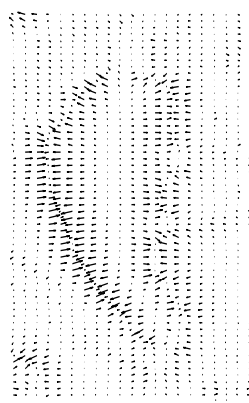

(b)

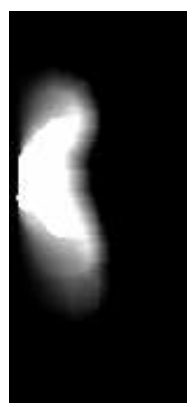

(c)

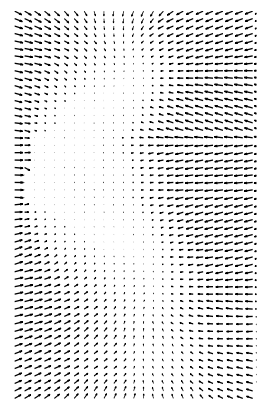

(d)

Fig. 3. Fusion of heterogeneous information in a deformable model. This example presents the knowledge used to segment the caudate nucleus by combining a deformable model with spatial relations. (a) Edge map corresponding to the contour of the caudate nucleus. (b) Data term derived from the previous edge map, denoted by $\mathbf{F}_{C}$ in Equation 2. In this case, a Gradient Vector Flow [24] is used to compute the force (for visualization purpose, a zoom has been performed on a part of the previous image). (c) Spatial relation corresponding to the conjunctive fusion of "near" and "lateral to the lateral ventricle". (d) Force corresponding to the previous spatial relation combination, denoted by $\mathbf{F}_{R}$ in Equation 2 (for visualization purpose, a zoom as well as a 1/2 undersampling has been performed).
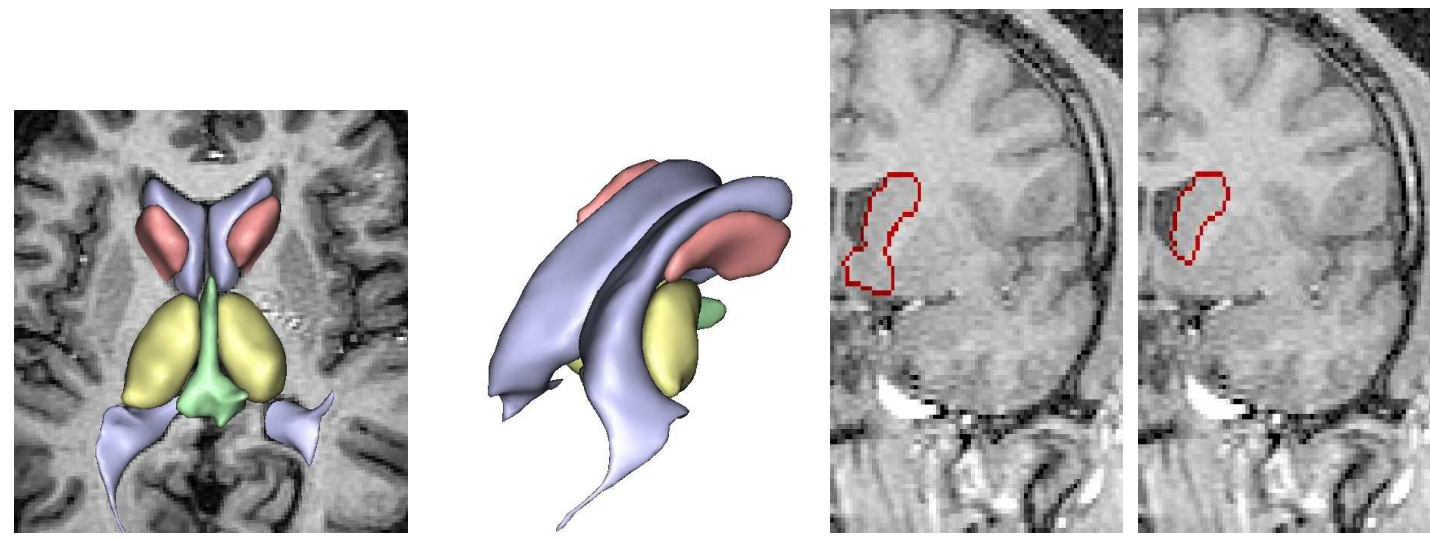

Fig. 4. Segmentation and recognition results obtained for the lateral ventricles, third ventricle, caudate nuclei and thalami by integrating spatial relations in 3D deformable models. Illustration of the importance of spatial relations in the deformable model: in the case of caudate nucleus, the force derived from spatial relations prevents the model to grow below the lower limit of the structure (third image: result obtained without this force, fourth image: with this force).

\section{Conclusion}

As illustrated in this paper, the semi-qualitative fuzzy set framework presents interesting features both for knowledge representation (of spatial relations, of imprecision existing both in the objects and in the relations), and for reasoning and recognition. We have also shown the usefulness of fuzzy mathematical 
morphology in this context. We paid attention in particular at the fusion step, which benefits from the high flexibility provided by the fuzzy fusion operators. Fusion of spatial relationships is proved to be useful in model-based recognition procedures, either global or progressive ones, and can be elegantly merged with powerful segmentation techniques. This work opens new perspectives for spatial reasoning under imprecision.

\section{References}

[1] B. J. Kuipers, T. S. Levitt, Navigation and Mapping in Large-Scale Space, AI Magazine 9 (2) (1988) 25-43.

[2] I. Bloch, Information Combination Operators for Data Fusion: A Comparative Review with Classification, IEEE Transactions on Systems, Man, and Cybernetics 26 (1) (1996) 52-67.

[3] D. Dubois, H. Prade, R. Yager, Merging Fuzzy Information, in: J. Bezdek, D. Dubois, H. Prade (Eds.), Handbook of Fuzzy Sets Series, Approximate Reasoning and Information Systems, Kluwer, 1999, Ch. 6.

[4] D. Dubois, H. Prade, Fuzzy Sets and Systems: Theory and Applications, Academic Press, New-York, 1980.

[5] I. Bloch, Fusion of Numerical and Structural Image Information in Medical Imaging in the Framework of Fuzzy Sets, in: P. S. et al. (Ed.), Fuzzy Systems in Medicine, Series Studies in Fuzziness and Soft Computing, Springer Verlag, 2000, pp. 429-447.

[6] M. Nachtegael, E. E. Kerre, Classical and Fuzzy Approaches towards Mathematical Morphology, in: E. E. Kerre, M. Nachtegael (Eds.), Fuzzy Techniques in Image Processing, Studies in Fuzziness and Soft Computing, Physica-Verlag, Springer, 2000, Ch. 1, pp. 3-57.

[7] I. Bloch, Fusion of Spatial Relationships for Guiding Recognition, with Examples in Brain Structure Recognition, in: International Conference on Advances in Pattern Recognition ICAPR 2003, Calcutta, India, 2003, pp. 25-30.

[8] I. Bloch, H. Maître, Fuzzy Mathematical Morphologies: A Comparative Study, Pattern Recognition 28 (9) (1995) 1341-1387.

[9] I. Bloch, Mathematical Morphology and Spatial Relationships: Quantitative, Semi-Quantitative and Symbolic Settings, in: L. Sztandera, P. Matsakis (Eds.), Applying Soft Computing in Defining Spatial Relationships, Physica Verlag, Springer, 2002, pp. 63-98.

[10] I. Bloch, On Fuzzy Distances and their Use in Image Processing under Imprecision, Pattern Recognition 32 (11) (1999) 1873-1895. 
[11] I. Bloch, Fuzzy Relative Position between Objects in Image Processing: a Morphological Approach, IEEE Transactions on Pattern Analysis and Machine Intelligence 21 (7) (1999) 657-664.

[12] D. Dubois, H. Prade, A Review of Fuzzy Set Aggregation Connectives, Information Sciences 36 (1985) 85-121.

[13] R. R. Yager, Connectives and Quantifiers in Fuzzy Sets, Fuzzy Sets and Systems 40 (1991) 39-75.

[14] I. Bloch, H. Maître, M. Anvari, Fuzzy Adjacency between Image Objects, International Journal of Uncertainty, Fuzziness and Knowledge-Based Systems 5 (6) (1997) 615-653.

[15] C. Xu, D. L. Pham, J. L. Prince, Medical Image Segmentation Using Deformable Models, in: J. M. Fitzpatrick, M. Sonka (Eds.), Handbok of Medical Imaging, SPIE Press, 2000, pp. 129-174.

[16] J. Montagnat, H. Delingette, N. Ayache, A Review of Deformable Surface: Topology, Geometry and Deformation, Image and Vision Computing 19 (2001) 1023-1040.

[17] O. Colliot, O. Camara, R. Dewynter, I. Bloch, Description of Brain Internal Structures by Means of Spatial Relations for MR Image Segmentation, in: SPIE Medical Imaging, CA, USA, 2004.

[18] B. Bouchon-Meunier, M. Rifqi, S. Bothorel, Towards General Measures of Comparison of Objects, Fuzzy Sets and Systems 84 (2) (1996) 143-153.

[19] A. Perchant, I. Bloch, Fuzzy Morphisms between Graphs, Fuzzy Sets and Systems 128 (2) (2002) 149-168.

[20] A. Perchant, C. Boeres, I. Bloch, M. Roux, C. Ribeiro, Model-based Scene Recognition Using Graph Fuzzy Homomorphism Solved by Genetic Algorithm, in: GbR'99 2nd International Workshop on Graph-Based Representations in Pattern Recognition, Castle of Haindorf, Austria, 1999, pp. 61-70.

[21] E. Bengoetxea, P. Larranaga, I. Bloch, A. Perchant, C. Boeres, Inexact Graph Matching by Means of Estimation of Distribution Algorithms, Pattern Recognition 35 (2002) 2867-2880.

[22] R. Cesar, E. Bengoetxea, I. Bloch, Inexact Graph Matching using Stochastic Optimization Techniques for Facial Feature Recognition, in: International Conference on Pattern Recognition ICPR 2002, Vol. 2, Québec, 2002, pp. 465468.

[23] I. Bloch, T. Géraud, H. Maître, Representation and Fusion of Heterogeneous Fuzzy Information in the 3D Space for Model-Based Structural Recognition - Application to 3D Brain Imaging, Artificial Intelligence Journal 148 (2003) $141-175$.

[24] C. Xu, J. L. Prince, Snakes, Shapes and Gradient Vector Flow, IEEE Transactions on Image Processing 7 (3) (1998) 359-369. 